\title{
Dank an Elmar Mittler
}

https://doi.org/10.1515/bfp-2021-1001

Sehr geehrter Herr Professor Mittler, lieber Elmar, seit der Gründung der Zeitschrift BIBLIOTHEK - Forschung und Praxis im Jahr 1977 bist Du ihr leitender, verantwortlicher Herausgeber. Du hast sie in mehr als vier Jahrzehnten gemeinsam mit den anderen Herausgebern zu einer der führenden deutschsprachigen Bibliothekszeitschriften entwickelt. Bis zum heutigen Tag erfüllt die Zeitschrift das bereits im ersten Heft formulierte programmatische Ziel, Publikationsort für neue Forschungen zum Bibliothekswesen und zur intensiven Diskussion von Forschungsansätzen zu sein. In der Rückschau ermöglichen die Themenschwerpunkte der einzelnen Jahrgänge einen konzisen Überblick über mehr als vier Jahrzehnte Innovationen im Bibliothekswesen, und sie weisen dabei weit über den deutschen Sprachraum hinaus. Zuletzt war es Dir ein besonderes Anliegen, die Zeitschrift noch als leitender Herausgeber in ein neues Open-Access-Modell zu überführen und mit „Subscribe to Open“ erneut einen innovativen Weg innerhalb der deutschen Publikationslandschaft $\mathrm{zu}$ gehen.

Nun, da Du Dich aus der Rolle des leitenden Herausgebers zurückziehst, müssen wir uns schweren Herzens daran gewöhnen, dass die jährlichen Herausgebertreffen im Archivraum des Verlages in der Genthiner Straße in Berlin nicht mehr von Dir in Deiner entschiedenen, ergebnisorientierten und stets produktiven Art geleitet werden. Für den Verlag können wir sagen, dass wir alle sehr froh sind, dass Du dem Herausgeberkreis der Zeitschrift auch weiterhin erhalten bleiben wirst und wir auf Deine Ideen und Kontakte auch künftig nicht werden verzichten müssen.

Deine einzigartige Bedeutung für das nationale und internationale Bibliothekswesen wurde 2020 anlässlich Deines 80. Geburtstags in einem Symposium zum Thema Transfer und Transformation. Bibliotheken als Vermittler im globalen Kontext eindrücklich dargestellt.

Dem Verlag bist Du über all die Jahrzehnte in zahlreichen Projekten verbunden gewesen. Aus den vielen Veröffentlichungen ragt das Jahrhundertprojekt der Mikrofiche-Ausgabe der Bibliotheca Palatina heraus. Anlässlich Deines 75. Geburtstags 2016 durften wir Dir eine Festschrift mit dem für Dein Wirken programmatischen Titel Bibliothek der Zukunft. Zukunft der Bibliothek widmen. Der Titel fasst sehr gut Dein jahrzehntelanges Wirken für eine Bibliothek der Zukunft sowie Deine Überlegungen und Initiativen für die Zukunft der Bibliothek zusammen, die Du als Autor, Forscher, Herausgeber und Lehrer bis auf den heutigen Tag aktiv gestaltest.

Lieber Elmar, wir danken sehr für die langjährige fruchtbare und wegweisende Zusammenarbeit mit Dir! Wir alle kennen Dich als kritischen Fragensteller und Ideengeber, und wir hoffen sehr, dass Du das in der neuen Herausgeberkonstellation noch lange bleiben wirst. Wir wünschen Dir persönlich alles Gute und freuen uns auf die nächsten gemeinsamen Jahre: Ad multos annos!

Manuela Gerlof, Claudia Heyer, Florian Hoppe und Martin Rethmeier 